

DiVA - Digitala Vetenskapliga Arkivet http://umu.diva-portal.org

This is an article published in Journal of Chemical Physics

Citation for the published paper:

Ove Andersson, Gyan P Johari, H Suga

An ice phase of lowest thermal conductivity

Journal of Chemical Physics, 2004 Vol. 120, Issue 20: 9612-9617

[URL: http://dx.doi.org/\#\#\#\#\#/\#\#\#\#\#\#] 


\title{
An ice phase of lowest thermal conductivity
}

\author{
Ove Andersson \\ Department of Physics, Umeå University, S-901 87 Umeå, Sweden \\ G. P. Joharia) \\ Department of Materials Science and Engineering, McMaster University, Hamilton, ON. L8S-4L7, Canada \\ H. Suga \\ Solid State Laboratory, Minoo 4-17-39, Osaka 562-0001, Japan
}

(Received 12 January 2004; accepted 19 February 2004)

\begin{abstract}
On pressurizing at temperatures near $130 \mathrm{~K}$, hexagonal and cubic ices transform implosively at 0.8-1 GPa. The phase produced on transformation has the lowest thermal conductivity among the known crystalline ices and its value decreases on increase in temperature. An ice phase of similar thermal conductivity is produced also when high-density amorphous ice kept at $1 \mathrm{GPa}$ transforms on slow heating when the temperature reaches $\sim 155 \mathrm{~K}$. These unusual formation conditions, the density and its distinguished thermal conductivity, all indicate that a distinct crystal phase of ice has been produced. (C) 2004 American Institute of Physics. [DOI: 10.1063/1.1703523]
\end{abstract}

\section{INTRODUCTION}

There are at least 13 crystalline forms of solid water. Ten of these, namely, ices II, III, IV, V, VI, VII, VIII, IX, X, and $\mathrm{XII}$, are produced at high pressures ${ }^{1}$ and three, hexagonal and cubic ices and ice XI, are produced at ambient pressure. There are also four amorphous forms of solid water, ${ }^{1,2}$ (i) glassy water and the vapor-deposited solid water, ${ }^{1}$ (ii) highdensity amorph (HDA), ${ }^{1,2}$ (iii) low-density amorph (LDA), ${ }^{1,2}$ and (iv) a very high-density amorph (VHDA). ${ }^{3}$ Thus the phase diagram of water is uniquely rich: it contains a variety of metastable and stable crystalline solids and incorporates features of both reversible and irreversible solid-solid transformations.

While the crystalline phases of water have been unequivocally established, the amorphous states have not been so. For example, it has been thought that there may be a first-order (reversible) transition between HDA and LDA, and this has led to the inference that there may be two states of liquid water, separated by a pressure-temperature phase boundary. This subject has been comprehensively reviewed recently ${ }^{4,5}$ and the possibility of the transition examined in context of the known results, but the issue of such a transition has remained unresolved. Ex situ performed neutron and x-ray-diffraction ${ }^{6-8}$ and Raman spectroscopy studies ${ }^{9}$ have shown that when HDA and LDA are kept at a fixed temperature at ambient pressure, amorphous solids of continuously different structures are formed. But still there are differences in the interpretation, as to how much of that change is due to structural relaxation of these phases and how much from actual HDA to LDA transformation. Koza et al. ${ }^{7}$ have concluded that their results "meet the conditions for a first-order transition" (between LDA and HDA). However, the view that there may be a first-order transition ${ }^{10}$ has now been revised and it has been stated, "In this respect, the LDA-HDA

a)Electronic mail: joharig@mcmaster.ca transformation observed in numerical simulation [7-9] should not be taken as proof of the existence of a liquidliquid transition." 11 The above mentioned observations ${ }^{6-9}$ and revision ${ }^{11}$ seem to call for reconsideration of our understanding of water's amorphous solids.

As part of our extensive study of the conditions for formation of HDA by pressurizing hexagonal ice (ice Ih), we had critically examined ${ }^{12}$ the results that had been taken to indicate a first-order transition between HDA and LDA. We had found that experimental data in fact did not show the reversibility of the HDA-LDA transition at a fixed temperature and pressure. Remarkably, our studies ${ }^{12}$ also showed that the state formed by pressurizing hexagonal ice at $\sim 130$ $\mathrm{K}$ continued to transform and/or to densify at a fixed pressure. This latter observation led to the conclusion that there may also be a continuity of amorphous states between those of HDA and VHDA. ${ }^{12}$ Therefore instead of only three amorphs that form via the pressure amorphization of hexagonal and cubic ices, a virtually infinite number of continuously distinct amorphs (varying in structure and properties between those of VHDA and LDA) can form via the pressure amorphization of these ices.

Here we report a detailed study of the conditions for formation of the high-density amorph by pressurizing hexagonal ice (ice Ih) and cubic ice (ice Ic). We find that at a relatively high temperatures of $\sim 130 \mathrm{~K}$, partially amorphized ice Ih and Ic spontaneously transform to a denser solid whose thermal conductivity is lowest amongst the known ice phases, and its magnitude decreases on heating, as is expected for a typical crystal phase. We also find that a solid of the same thermal conductivity forms when the highdensity amorph kept at a fixed pressure of $1 \mathrm{GPa}$ is heated from $130 \mathrm{~K}$ to a temperature above $155 \mathrm{~K}$, thus providing two sets of pressure-temperature conditions for producing this phase. The massive high pressure apparatus used for such measurements prevented us from observing the diffraction pattern of this ice phase. Also the slow heat transfer to 
the sample prevented us from quenching it to $77 \mathrm{~K}$, which is required for performing ex situ diffraction studies at ambient pressure. Since most high-pressure phases of ice had been discovered in the earlier part of the last century without diffraction studies, structural identification of the phase formed here does not seem essential at this stage. When more sophisticated equipment become available, the crystalline ice phase of lowest thermal conductivity, which is formed here in conditions different from those known for other ices, can be identified.

\section{EXPERIMENTAL METHODS}

Thermal conductivity $\kappa$ of the ice phases was used as a distinctive property for detecting different phase transformations, and for identifying the various ice phases. The measurement assembly consisted of a 37-mm internal diameter, 25-ml capacity Teflon vessel with a built-in 0.1-mm-diameter $\mathrm{Ni}$-wire ring as hot-wire probe and a thermocouple as temperature sensor. It was tightly inserted in a $45-\mathrm{mm}$ internal diameter high-pressure cylinder. The vessel was filled with $\sim 20 \mathrm{ml}$ of water purified by using Milli-Q ${ }^{\circledR}$ Ultrapure WaterSystems, sealed with a 5-mm-thick tightly fitting Teflon cover and the piston inserted. The assembly was pressurized to $0.07 \mathrm{GPa}$ and then cooled at an initial rate of about 0.5 $\mathrm{K} / \mathrm{min}$ to the desired temperature $T$ by means of a built-in helium cryostat. After the water froze to ice Ih, the pressure $p$ on the sample was raised (or lowered) at the desired rate by means of computer-controlled hydraulic system that moved the piston inside the cylinder. Ice Ic was made by depressurizing a high pressure ice phase to $p<0.07 \mathrm{GPa}$, and then slowly heating the sample. Its formation was monitored by measuring the thermal conductivity as before. ${ }^{12,13}$

In a typical experiment, samples of ices Ih and Ic were maintained at a fixed $T$ at several temperatures in the 120 $130-\mathrm{K}$ temperature range. At a fixed temperature, the pressure was increased at the rate of $0.1 \mathrm{GPa} / \mathrm{h}$. Cooling and heating of the samples were done at the rate of $5-30 \mathrm{~K} / \mathrm{h}$. Because of the exceptionally slow rates of the pressure and temperature changes, our results do contain the consequences of the long time ( $48-72 \mathrm{~h})$ taken to prepare and to in situ study the high-density amorph and other ices that could form. Also the sample size here is much larger than used in the earlier studies. The quantities needed for determining $\kappa$ were measured in real time and the temperature and pressure were automonitored by computer controls continuously during the course of an experiment, which itself took several days of $24 \mathrm{~h}$ operation. The accuracy of data is: $\pm 0.05 \mathrm{GPa}$ for $p$ (at $1 \mathrm{GPa}$ and $100 \mathrm{~K}$ ), $\pm 0.3 \mathrm{~K}$ for $T$ and $\pm 3 \%$ for $\kappa$.

\section{RESULTS AND DISCUSSION}

Ice Ih was first pressurized at an approximately fixed $T$, and its $\kappa$ was measured continuously in real time. The plot of its $\kappa$ against $p$ is provided (circles) in Fig. 1. The inverted sigmoid-shape decrease in $\kappa$, which is observed here over the $0.75-0.95$-GPa range, shows, as before, ${ }^{12,13}$ that ice Ih is beginning to amorphize. [To avoid confusion, only the amorph formed by pressurizing ice Ih at $77 \mathrm{~K}$ (Refs. 1 and 2)

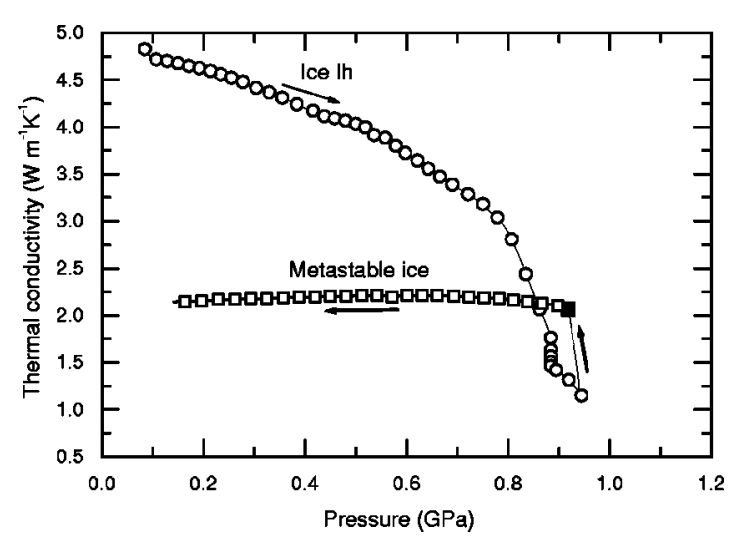

FIG. 1. Thermal conductivity of ice Ih is plotted against pressure at $\sim 130$ $\mathrm{K}$, before and during its transformation to the high-density amorph (circles). (The slope-discontinuity in the $0.4-0.5-\mathrm{GPa}$ range is caused by slow temperature changes during pressurization, and the vertical decrease at 0.88 GPa occurred when $p$ was kept fixed.) The slight rise of $\kappa$ in the 0.95 to 0.60 $\mathrm{GPa}$ range after transformation to the metastable ice is at least partly due to decreasing $T$ from 128 to $113 \mathrm{~K}$ during depressurization, but may also be caused when any residual high-density amorph had transformed.

is henceforth called HDA.] As the pressure was continuously increased we expected that the sample's $\kappa$ could decrease to the characteristic low value of $0.70 \mathrm{~W} \mathrm{~m}^{-1} \mathrm{~K}^{-1}$ for the highdensity amorph at a pressure of $1.1 \mathrm{GPa} .{ }^{13}$ But this did not occur. Instead, a loud implosive sound came from the pressure vessel, the pressure decreased instantaneously from 0.95 to $0.85 \mathrm{GPa}$. Simultaneously, $\kappa$ increased from 1.15 to 2.1 $\mathrm{W} \mathrm{m}{ }^{-1} \mathrm{~K}^{-1}$ and the temperature increased from 128 to 155 $\mathrm{K}$. The changes observed in the pressure and $\kappa$ values are shown by the filled squares in Fig. 1. The pressure and temperature were autorestored to pre-implosion values and, thereafter, the pressure on the sample was decreased to 0.2 $\mathrm{GPa}$ at a controlled rate and its $\kappa$ was continuously measured. These $\kappa$ values are plotted as open squares in Fig. 1.

During the course of programmed increasing of the pressure on ice Ih and Ic at 120-130 K several samples imploded similarly at $0.85-1.0 \mathrm{GPa}$. The changes observed in the pressure, temperature, and $\kappa$ on these implosions were closely similar to those described in connection with Fig. 1. The implosion, i.e., a decrease in volume, and the attendant changes in the pressure and temperature indicate that an exothermic transformation to a denser phase has occurred. We stress that the piston in our pressure assembly moved freely and continuously, which is in contrast to those in earlier studies at $77 \mathrm{~K},{ }^{2,14,15}$ in which the piston frequently jammed by increased friction between it and the pressure vessel. Therefore there was no sudden release of the piston after overcoming of this friction, which could have increased the temperature. We conclude that the decrease in volume and the increase in temperature did not cause conversion of the highdensity amorph to a still denser crystalline ice phase. Instead, they were the consequences of this conversion.

The pressure on the ices thus formed was decreased from the autorestored pre-implosion value, which varied for different samples, to $\sim 0.1$ or $0.15 \mathrm{GPa}$ at a controlled rate in the range of $\sim 0.05-0.1 \mathrm{GPa} / \mathrm{h}$. Simultaneously, the samples were cooled to $\sim 110 \mathrm{~K}$, at the rate of $\sim 3-6 \mathrm{~K} / \mathrm{h}$ rate. Thereafter, the samples were heated at $15 \mathrm{~K} / \mathrm{h}$, while their $\kappa$ was 


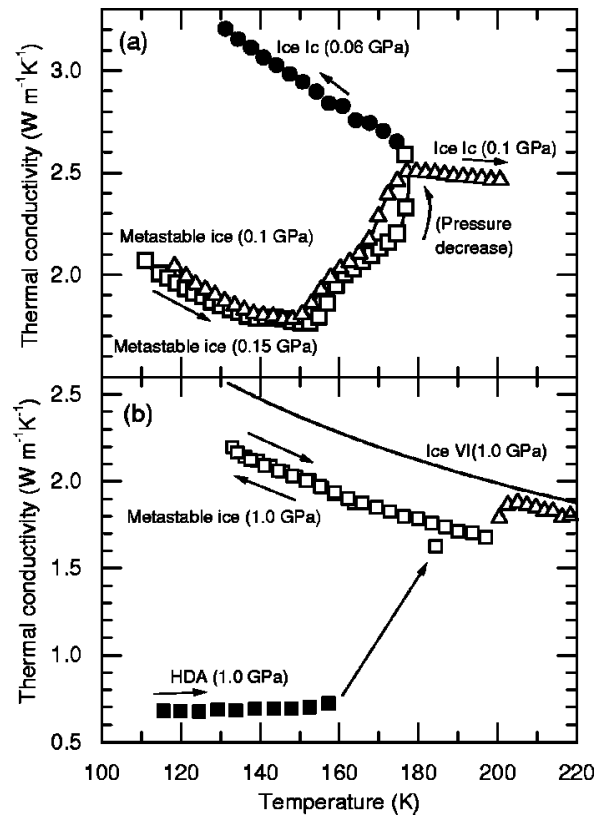

FIG. 2. (a) Thermal conductivity of the metastable ice is plotted against $T$ at the indicated $p$. The increase in $\kappa$ beginning at $\sim 150 \mathrm{~K}$ is due to transformation to another state and finally to ice Ic, both on continued heating and on cooling from $\sim 175 \mathrm{~K}$. (b) Thermal conductivity of the high-density amorph at $1 \mathrm{GPa}$ heated from $115 \mathrm{~K}$. Solid squares are for the high-density amorph, open squares are for the metastable ice phase (cooling and heating), and triangles are for ice VI. The solid line is from Ref. 16.

measured continuously. The plots of $\kappa$ against $T$ for two such samples at pressures of 0.1 and $0.15 \mathrm{GPa}$ are presented in Fig. 2(a). It is evident that $\kappa$ decreases with increase in $T$ initially. This is followed by a small sigmoid-shape rise in $\kappa$ over the temperature range of $150-165 \mathrm{~K}$ in one case, and of $152-170 \mathrm{~K}$ in the other. The sample kept at 0.1 GPa was continuously heated and its $\kappa$ (denoted by triangles) showed a second sigmoid-shape increase and finally a slight decrease at $\sim 175 \mathrm{~K}$. The sample kept at $0.15 \mathrm{GPa}$ was depressurized to $0.06 \mathrm{GPa}$ at a temperature of $\sim 175 \mathrm{~K}$ and then cooled. Its $\kappa$ (shown by squares) increased on cooling, as shown by dots in Fig. 2(a). A comparison of the magnitude of its $\kappa$ against that of other ices ${ }^{16}$ showed that ice Ic had formed. This is labeled accordingly in Fig. 2(a). The first sigmoid-shape increase in $\kappa$ for the two samples, one at $0.1 \mathrm{GPa}$ and the second, at $0.15 \mathrm{GPa}$ in Fig. 2(a), indicates that the sample has transformed to a mixture of several ice phases, or to a phase containing stacking faults prior to its full conversion to ice Ic. (Although not shown here, it is worth noting that when this ice Ic was subsequently pressurized at $128 \mathrm{~K}$, it also underwent an implosive transition but at a pressure of $1.08 \mathrm{GPa}$ and produced a phase with approximately the same $\kappa$ value as given for the metastable ice in Fig. 1.)

In several of our experiments, increase in the pressure on samples of ice Ih kept in the temperature range of 120-130 $\mathrm{K}$, completely transformed the samples to a high-density amorph, i.e., no implosion occurred during the pressurization. When this high-density amorph was in situ heated at 1 $\mathrm{GPa}$, its thermal conductivity increased on heating very slowly first, and then abruptly at $\sim 159 \mathrm{~K}$. An exothermic effect, i.e., temperature rise, occurred in the 155-157-K range. This indicates that a spontaneous transformation of the amorph had occurred to another phase of ice. Although $\kappa$ increased abruptly when this transformation occurred, there was no significant decrease in pressure or volume.

Figure 2(b) shows the results from one such experiment on the high-density amorph formed. The amorph had been produced by pressurizing ice Ih at $130 \mathrm{~K}$ to $1.2 \mathrm{GPa}$. The pressure was decreased from 1.2 to $1.0 \mathrm{GPa}$ and the temperature from 130 to $115 \mathrm{~K}$. The sample was then kept at a fixed pressure of $1.0 \mathrm{GPa}$ and heated at the rate of $18 \mathrm{~K} / \mathrm{h}$. When the temperature reached $\sim 155 \mathrm{~K}$, the sample spontaneously transformed and its $\kappa$ increased by a factor of 3 as indicated by the large arrow. The transformed sample was subsequently cooled. Its $\kappa$ increased and reached within $\sim 5 \%$ of the $\kappa$ value obtained here for the distinct phase which had formed by implosive transformation of ice Ih and Ic, as is shown (metastable ice) in Figs. 1 and 2(a).

A subsequent experiment was performed for measuring the change in the properties of the ice phase obtained by heating the high-density amorph at $\sim 1.0 \mathrm{GPa}$. In this experiment, the ice phase thus obtained was cooled while its $\kappa$ was being measured in real time. This $k$ value is plotted against the temperature in Fig. 2(b) and is shown by squares and an arrow indicating the cooling. [As in Figs. 1 and 2(a), this sample has been cautiously labeled as a "metastable ice," mainly because its phase boundary is not known, and it is not certain that it has a thermodynamic region of stability in the $p-T$ diagram of water.] The sample was then heated, and its $\kappa$ is also plotted in Fig. 2(b) and shown by an arrow next to the square notation for the data points. It is evident that its $\kappa$ value retraces the curve obtained on cooling, thereby indicating the reproducibility of the measured values and the stability of the state of the sample.

On heating the distinct phase at $1 \mathrm{GPa}$ beyond $195 \mathrm{~K}$, its $\kappa$ showed a small sigmoid-shape increase beginning at $\sim 198$ $\mathrm{K}$, thus indicating a transformation to another ice phase which continued until the temperature reached $\sim 205 \mathrm{~K}$ during this heating. The final $\kappa$ value was compared against the $\kappa$ values known for other high pressure crystalline ices as given in Ref. 16, and it was found that this value is within $4 \%$ of the $k$ value for ice VI at $1 \mathrm{GPa}$. For convenience of comparison, a smooth curve has been drawn in Fig. 2(b) to show the known variation of $\kappa$ of ice VI taken from Ref. 16. We conclude that slow heating of the distinct phase, which had been formed here by heating the amorph at $1.0 \mathrm{GPa}$, transforms the distinct (metastable ice) phase to ice VI. In summary, a metastable crystalline ice phase of lowest $\kappa$ formed in two ways, (i) implosively during the amorphization of ice Ih and ice Ic at $130 \mathrm{~K}$, and (ii) on heating the high-density amorph at $1 \mathrm{GPa}$ from $130 \mathrm{~K}$ when the temperature reached $\sim 155 \mathrm{~K}$. On heating at a fixed pressure of $1 \mathrm{GPa}$, this metastable phase began to transform to ice VI when the temperature reached $198 \mathrm{~K}$.

In these studies, more than ten sets of experiments showed the same transformations of ice Ih or Ic occurring in the same pressure range. But the exact pressure of implosion varied with the temperature, pressure, thermal history, microstructural details, and contaminants of the sample. It is evident that this transformation basically differs from the vari- 


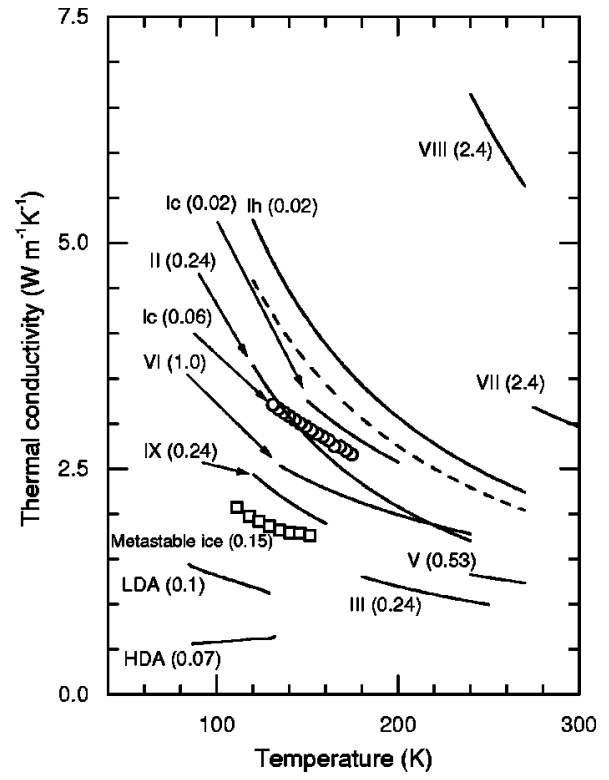

FIG. 3. Thermal conductivity of the metastable phase and of several crystalline ices is plotted against $T$ at the indicated $p$. For comparison, the values for the high-density amorph and LDA taken from Ref. 13 are included. The data for the other ices are from Ref. 16. The dashed line is for $\kappa \propto T^{-1}$.

ous transformations observed earlier, such as those where ice XII formed via HDA as a result of a shock wave. ${ }^{17}$

It should also be stressed that the structure and properties of an amorph formed at high pressures vary with the $p$ and $T$ conditions by which it has been produced. ${ }^{2}$ For example, HDA produced by slowly increasing and carefully controlling the pressure on ice Ih at $77 \mathrm{~K}$ and keeping the pressure at $1.7 \mathrm{GPa}$ for a long duration was found to have a density as high as $\sim 1.30 \mathrm{~g} / \mathrm{ml}$ at ambient pressure and 77 K. ${ }^{18}$ Also, it has not been widely recognized that HDA itself had shown structural differences in some of the earliest diffraction studies. For example, the position of the first sharp $\mathrm{x}$-ray diffraction peak in the structure factor of HDA is at $2.10 \AA^{-1}$ in the study in Ref. 14 , and at $2.25 \AA^{-1}$ in the study in Ref. 6. Therefore it is likely that the high-density amorph produced here, by allowing a longer time for pressure to increase to $\sim 1 \mathrm{GPa}$ at a higher temperature of $\sim 130 \mathrm{~K}$, would not be the same as the HDA produced by relatively rapidly increasing the pressure to $\sim 1 \mathrm{GPa}$ and 77 $\mathrm{K}^{2,3,14,15,17,19-21}$ Loerting et al. ${ }^{3}$ have shown that when HDA at a pressure in the range of $\sim 0.8-1.9 \mathrm{GPa}$ is heated from 77 to $165 \mathrm{~K}$ it gradually densifies by $\sim 9 \%$ to a very highdensity amorph (VHDA). But the high-density amorph here at a fixed pressure of $1 \mathrm{GPa}$ shows, in Fig. 2(b), that on gradual heating it abruptly transforms at $\sim 155 \mathrm{~K}$. In their later studies, ${ }^{22}$ however, they also found that heating of HDA at $\sim 1.09 \mathrm{GPa}$ transforms it to ice XII at $175 \mathrm{~K}$, after it has densified considerably.

For the purpose of comparing the $\kappa$ value of the metastable crystalline ice phase produced here against the $\kappa$ value of other ices as well as for placing this distinct phase in context with other ices, we summarize the thermal conductivity data of HDA (the then labeled high-density amorph), LDA, ${ }^{13}$ and of crystalline phases of ice ${ }^{16}$ in Fig. 3 and also show the variation in their thermal conductivity with the temperature. The range of temperature and pressure for which data have been provided is limited by both the thermodynamic stability range of the ices and the limits of thermal conductivity measurements on them. A comparison of the plots clearly shows that the ice phase formed here has the lowest thermal conductivity among all ices in their stability range.

Although it is not the main objective of this paper, we discuss the structural aspects that may cause a low thermal conductivity of the distinct crystalline ice phase produced here. First, we recall that any change that favors strong phonon-phonon umklapp scattering, which are caused by anharmonic lattice vibrations, decreases the thermal conductivity. However, the low value of thermal conductivity of the ice phase produced here is most likely not due to an exceptionally large anharmonic lattice vibrations in comparison with similar contributions in other ices. We attribute this low value to microstructural disorder in addition to any proton disorder in the structure of this distinct crystal phase.

Some idea of the role of anharmonicity of phonons may be gained from the following consideration. Earlier studies ${ }^{23}$ of thermal conductivity of both ice Ih before and after its proton ordering to ice XI have shown that anharmonicity of phonons decreases on proton ordering of ice Ih to ice XI, or alternatively increases on proton disordering of ice XI to ice Ih. But the increase in the anharmonicity of phonons that occurs on proton disordering is not sufficient to decrease $\kappa$ by more than $\sim 20 \%$. Moreover, the increase in umklapp scattering consequent to increase in anharmonicity does not affect the temperature dependence of $\kappa$, unless some other scattering process is also being significant in limiting $\kappa$. In fact, the temperature dependence of $\kappa$ can be a guide for determination of the main cause for phonon scattering. To make use of this, we determine the temperature dependence of thermal conductivity, i.e., $(d \ln \kappa / d \ln T)_{0.15 \mathrm{GPa}}$ of the phase produced here from our data in Fig. 2(a). Its value is -0.6. This $(d \ln \kappa / d \ln T)_{0.15 \mathrm{GPa}}$ value is significantly more than the value of -1.0 that is expected at temperatures above the Debye temperature for a crystal in which umklapp scattering strongly dominates. Therefore the value of $(d \ln \kappa / d \ln T)_{0.15 \mathrm{GPa}}=-0.6$ for the distinct crystal phase shows that umklapp scattering is probably dominant, but that there should also be another scattering source, with weaker temperature dependence, which enhances the phonon scattering.

In a further analysis, we combine the results provided in Figs. 2(a) and 2(b) and obtain the pressure coefficient of the thermal conductivity, $(d \ln \kappa / d p)_{130 \mathrm{~K}}$. Its value is 0.15 $\mathrm{GPa}^{-1}$. Since a large lattice anharmonicity is likely to cause strong pressure dependence of $\kappa$, this low value of $(d \ln \kappa / d p)_{130 \mathrm{~K}}$ supports our above-mentioned interpretation that the low $\kappa$ value is not due to unusually strong phononphonon scattering. We conclude that these distinctions further demonstrate that the implosively formed ice phase is different from the known ones.

It is worth pointing out that except for ice Ic and ice XII, all stable and metastable phases of ice had been discovered by observing changes in their volume and in their dielectric properties at certain pressures and temperatures. The phases 
were then given Roman numbers, their phase boundaries established and the phase diagrams of water constructed, all without the aid of diffraction studies - and we recall that, in most cases, this had been done prior to the availability of the $\mathrm{x}$-ray-diffraction measurements. Even now, measurements of a macroscopic property are preferred for investigating phase transformations, mainly because unresolvable and subtle differences in the crystal structures can cause a large change in macroscopic properties. Our massive, high pressure assembly, which is kept under vacuum, does not allow in situ studies by diffraction methods. It also does not allow rapid cooling of the sample to $\sim 77 \mathrm{~K}$ at high pressures and subsequently extracting the sample at atmospheric pressure rapidly enough to avoid heating above $100 \mathrm{~K}$. Therefore $e x$ situ diffraction studies of an ice phase at ambient pressure could also not be performed in this study. It is worth reiterating that isothermal pressurization of ice Ih at $T>140 \mathrm{~K}$ can yield ice III and/or ice IX, ${ }^{20}$ but these phases are bulkier than HDA, ${ }^{1}$ and even bulkier than the high-density amorph formed here. The only phases whose $\kappa$ has not so far been studied are ice IV and ice XII, both of which are metastable. These two ice phases were originally obtained by compressing liquid water to a pressure within the region of thermodynamic stability of ice $\mathrm{V}{ }^{1}$

On slow heating at a high pressure, HDA does not transform to LDA. Instead it crystallizes to various high pressure phases of ices, in some cases to a mixture of two highpressure phases which remains kinetically stable for a period long enough for ex situ studies by x-ray diffraction of the recovered phase at ambient pressure ${ }^{17,21,22}$ and in situ studies by neutron diffraction at high pressures. ${ }^{19,20}$ Klotz et al. have reported ${ }^{19,20}$ that slow heating of HDA over a large pressure range $(0.6-1.5 \mathrm{GPa})$, crystallizes it to metastable proton disordered phases, and that ice XII is formed in the highpressure part of this range. Neutron-diffraction spectra in their Fig. 3 show $^{20}$ that on heating at a fixed pressure of 0.6-0.7 GPa, $\mathrm{D}_{2} \mathrm{O}$ HDA crystallized to a mixture of ice IV and XII at $165 \mathrm{~K}$ and that the mixture completely converted to ice IV at $210 \mathrm{~K}$. They also found that on heating in the 1-1.5-GPa range, $\mathrm{D}_{2} \mathrm{O}$ HDA crystallized to a mixture of ice VI and ice XII, and, “...that phase XII exists up to $\sim 208 \mathrm{~K}$ in essentially the same crystallographic form as grown from the liquid...." ${ }^{20}$ This is in contrast to the phase formed here that crystallizes at a lower temperature of $198 \mathrm{~K}$ to ice VI, as noted in Fig. 2(b). Klotz et al. ${ }^{20}$ recognized that small amounts of contaminant crystalline phases may act as seeds and may change the crystallization behavior. We recall that equilibrium pressure and temperature of transformation of ice phases for $\mathrm{D}_{2} \mathrm{O}$ differ from those of $\mathrm{H}_{2} \mathrm{O}$, and also that irreversible crystallization is often determined by the rate of molecular diffusion, which varies with pressure, and therefore on the heating rate of a solid. This aspect has been discussed in detail in a recent paper ${ }^{12}$ with particular reference to the irreversible conversion of HDA to LDA and the crystallization of LDA to ice Ic.

In our discussion of the implosively transformed phase, we have cautiously used the crystallization conditions of HDA as a guide. This HDA had been produced at a temperature and pressure different from those in our study, and the pressure in those studies ${ }^{14,15,19-21}$ had not been known with the same accuracy as here, and in some studies has not appear to be corrected for friction in the high-pressure assembly. (Klotz et al. ${ }^{20}$ estimated accuracy of $\sim 0.1 \mathrm{GPa}$ and Salzmann et al. ${ }^{21}$ did not state the accuracy in their pressure data.) There is also an additional effect due to the long time taken for pressurizing, cooling, and heating of the various intermediate and final states formed in our study. We also stress that the distinct (metastable ice) phase in our study had formed implosively and at pressure-temperature-time conditions sufficiently different from the conditions in which ice VI and/or ice XII had formed in the study by Klotz et al. ${ }^{19,20}$ and by Salzmann et al. ${ }^{21}$ Rapid heating of HDA at $0.81 \mathrm{GPa}$ has been known to transform it to ice XII and slow heating to ice IV. ${ }^{21}$ But in our study, the implosive transformation occurred at a fixed temperature of $\sim 130 \mathrm{~K}$, and it was not caused by heating. Even so, we do not rule out the possibility that ice IV and XII could have formed because no data on thermal conductivity of these ices are available for comparison. Koza et al., ${ }^{24}$ who pressurized ice Ih at $130 \mathrm{~K}$ at 0.5 $\mathrm{GPa} / \mathrm{min}$ to $1.8 \mathrm{GPa}$, produced XII. Moreover, it has also been found that heating of HDA at $\sim 0.84-1.09 \mathrm{GPa}$ crystallizes it to ice XII (sometimes mixed with VI), but not IV. ${ }^{20,22}$ Therefore we surmize that if a known ice phase were to have formed in our study, that phase, we cautiously speculate, could be ice XII. Until x-ray-diffraction studies have been performed, the question about its identity would remain open. For that reason we do not give a new name to the phase formed here, although we recall that in the 1960s, ice VIII (Ref. 25) and ice IX (Ref. 26) had been named only on the basis of their different dielectric properties; their x-raydiffraction studies were performed much later. ${ }^{1}$ We hope that the sintered diamond cell currently used as a pressure vessel for high-pressure studies by Klotz et al. ${ }^{19,20}$ or other highpressure diamond cells can be adapted for the purpose of studies under pressure-temperature-time conditions similar to ours. This would help in unequivocally identifying the distinct ice phase produced here.

It is also worth pointing out that the internal energy and structure of the ice phases measured in situ at high pressures are expected to differ from those of the same phases measured ex situ at ambient pressure. Since in situ studies are expected to provide direct information on the phase diagram of ices, without the need for extrapolating their metastability range, it is desirable to study changes in the properties of such phases at high pressures. For example, in situ transformation has been known to produce phases different from $e x$ situ transformations: A variety of high-pressure ice phases are produced when HDA crystallizes in situ at high pressures, ${ }^{19-23}$ but only ice Ic is produced when HDA crystallizes ex situ at ambient pressure.

The low thermal conductivity of the implosively produced ice phase has implications for planetary geology. It has been proposed that high-pressure crystalline phases may be produced by meteorite impact on the surface of ice satellites and icy planets. ${ }^{27}$ If the crystalline ice produced here were also to form on meteoritic impact in preference to other ices and were to remain at low temperatures, its low thermal conductivity will slow the rate of heat transfer from the interior 
of the planet to the ice surface. In this respect, we suggest that production of this distinct ice phase may be significant for planetary geology.

\section{ACKNOWLEDGMENTS}

We are grateful to the technical staff of Umea University for assistance. This research was supported by a grant from the Swedish Research Council. G.P.J. acknowledges NSERC of Canada's continued support for his general research.

${ }^{1}$ V. F. Petrenko and R. W. Whitworth, Physics of Ice (Oxford University Press, Oxford, 1999).

${ }^{2}$ O. Mishima, L. D. Calvert, and E. Whalley, Nature (London) 310, 393 (1984).

${ }^{3}$ T. Loerting, C. Salzmann, I. Kohl, E. Mayer, and A. Hallbrucker, Phys. Chem. Chem. Phys. 3, 5355 (2001).

${ }^{4}$ P. G. Debenedetti, J. Phys.: Condens. Matter 15, R1669 (2003).

${ }^{5}$ B. Guillot and Y. Guissani, J. Chem. Phys. 119, 11740 (2003).

${ }^{6}$ C. A. Tulk, C. J. Benmore, J. Urquidi, D. D. Klug, J. Neuefeind, B. Tomberli, and P. A. Egelstaff, Science 297, 1320 (2002).

${ }^{7}$ M. M. Koza, H. Schober, H. E. Fischer, T. Hansen, and F. Fujara, J. Phys.: Condens. Matter 15, 321 (2003).

${ }^{8}$ M. Guthrie, J. Urquidi, C. A. Tulk, C. J. Benmore, D. D. Klug, and J. Neuefeind, Phys. Rev. B 68, 184110 (2003).

${ }^{9}$ Y. Suzuki and O. Mishima, J. Phys. Soc. Jpn. 72, 3128 (2003).

${ }^{10}$ O. Mishima and H. E. Stanley, Nature (London) 392, 164 (1998).
${ }^{11}$ N. Giovambattista, H. E. Stanley, and F. Sciortino, Phys. Rev. Lett. 91, 115504 (2003).

${ }^{12}$ G. P. Johari and O. Andersson, J. Chem. Phys. 120, 6207 (2004).

${ }^{13}$ O. Andersson and H. Suga, Phys. Rev. B 65, 140201 (2002).

${ }^{14}$ L. Bosio, G. P. Johari, and J. Teixeira, Phys. Rev. Lett. 56, 460 (1986).

${ }^{15}$ A. Bizid, L. Bosio, A. Defrain, and M. Oumezzine, J. Chem. Phys. 87, 2225 (1987).

${ }^{16}$ R. G. Ross, P. Andersson, and G. Bäckström, J. Chem. Phys. 68, 3967 (1978).

${ }^{17}$ I. Kohl, E. Mayer, and A. Hallbrucker, Phys. Chem. Chem. Phys. 3, 602 (2001).

${ }^{18}$ G. P. Johari, J. Chem. Phys. 112, 8573 (2000); 113, 10412 (2000).

${ }^{19}$ S. Klotz, G. Hamel, J. S. Loveday, R. J. Nelmes, and M. Guthrie, ISIS, Rutherford Appleton Laboratory Report 11642, 2000-2001 (http:// www.isis.rl.ac.uk/isis2001/reports/11642.PDF).

${ }^{20}$ S. Klotz, G. Hamel, J. S. Loveday, R. J. Nelmes, and M. Guthrie, Z. Kristallogr. 218, 117 (2003).

${ }^{21}$ C. Salzmann, I. Kohl, T. Loerting, E. Mayer, and A. Hallbrucker, Can. J. Phys. 81, 25 (2003).

${ }^{22}$ T. Loerting, I. Kohl, C. Salzmann, E. Mayer, and A. Hallbrucker, J. Chem. Phys. 116, 3171 (2002).

${ }^{23}$ O. Andersson and H. Suga, Phys. Rev. B 50, 6583 (1994).

${ }^{24}$ M. M. Koza, H. Shoeber, T. Hansen, M. Toelle, and F. Fujara, Phys. Rev. Lett. 84, 4112 (2000).

${ }^{25}$ E. Whalley, D. W. Davidson, and J. B. R. Heath, J. Chem. Phys. 45, 3976 (1966).

${ }^{26}$ E. Whalley, J. B. R. Heath, and D. W. Davidson, J. Chem. Phys. 48, 4362 (1968)

${ }^{27}$ E. S. Gaffney and D. L. Matson, Icarus 44, 511 (1980). 